\section{Cahiers de Narratologie}

Analyse et théorie narratives

10.2 | 2001

La voix narrative

\title{
Le monologue intérieur dans l'œuvre de Juan Rulfo : une tromperie cathartique
}

\section{Marie-Agnès Palaisi-Robert}

\section{(2) OpenEdition}

\section{Journals}

Édition électronique

URL : http://journals.openedition.org/narratologie/10246

DOI : 10.4000/narratologie.10246

ISSN : 1765-307X

Éditeur

LIRCES

\section{Édition imprimée}

Date de publication : 1 janvier 2001

Pagination : 331-340

ISBN : 2914561032

ISSN : 0993-8516

\section{Référence électronique}

Marie-Agnès Palaisi-Robert, « Le monologue intérieur dans l'œuvre de Juan Rulfo : une tromperie cathartique », Cahiers de Narratologie [En ligne], 10.2 | 2001, mis en ligne le 01 janvier 2001, consulté le 11 juin 2020. URL : http://journals.openedition.org/narratologie/10246 ; DOI : https://doi.org/10.4000/ narratologie.10246 


\title{
LE MONOLOGUE INTÉRIEUR DANS L'GEUVRE DE JUAN RULFO : UNE TROMPERIE CATHARTIQUE.
}

\author{
Marie-Agnès PALAISI-ROBERT \\ Université de Montpellier III
}

Dans Pedro Páramo, les derniers instants du cacique sont décrits de la façon suivante :

«Después de unos cuantos pasos cayó, suplicando por dentro ; pero sin decir una sola palabra. Dio un golpe seco contra la tierra y se fue desmoronando como si fuera un montón de piedras. »1

Pedro Páramo meurt seul, sans plus aucun interlocuteur capable de lui renvoyer l'image du cacique invincible qu'il avait construite méthodiquement. Son pouvoir reposait sur un triangle inébranlable constitué par la violence, l'argent et la parole. Lorsque l'autorité physique et verbale ne suffit plus à faire respecter les ordres, le cacique achète le silence.

La parole, qu'elle soit autoritaire, silencieuse ou insidieuse, est au centre de cette stratégie de pouvoir. Celui qui a la parole est celui qui a le pouvoir. A la fin de sa vie, Pedro Páramo restait des journées entières prostré devant le chemin conduisant au cimetière, et son impossibilité de gémir pendant son agonie est le signe qu'il a perdu le contrôle de sa vie et de Comala.

Ce village est ainsi devenu le lieu du silence le plus lourd de sens et après la mort du cacique les langues se sont déliées, c'est-à-dire que s'opère une passation de pouvoir. La complexité de l'univers rulfien repose sur l'ambiguïté de cette parole silencieuse qui est indispensable au fonctionnement des systèmes autoritaires, mais qui menace aussi toujours ce dernier d'un possible dévoilement de ses fondations.

1 Juan RUlfo, Pedro Páramo, Facsímil de la edición de 1955, México : F.C.E., 1996, 156 p., p. 156 
Le monologue (intérieur) est le système d'organisation du récit qui structure tant les contes que le roman de Juan Rulfo. L'analyse de cette structuration dévoile une stratégie narrative particulière qui s'enracine dans une interprétation précise du fonctionnement des relations sociales dans un contexte répressif. La fonction et les particularités du monologue intérieur dans l'œuvre de Rulfo, qui est à la fois le signe de l'aliénation de l'individu et de son unique possibilité de libération, dévoile également une propension constante du récit à « l'engaño » à laquelle contribue cette parole à la fois silencieuse et prolifique.

Dans la plupart des contes de El llano en llamas, un personnage raconte sa propre histoire - « La Cuesta de las Comadres », "Macario », "Luvina » par exemple - ou une anecdote qu'il a directement ou indirectement vécue - «El día del derrumbe », "La herencia de Matilde Arcángel ». Souvent, le conte fait l'objet d'un aveu honteux ou criminel. Dans «Talpa », le frère du défunt Tanilo Santos avoue qu'il a entrepris le pélerinage à Talpa non pas tant dans l'espoir d'une guérison pour son frère que dans l'idée qu'un si long et si fatigant voyage pour l'infirme serait fatal. Sa confession est difficile, car ni lui ni sa belle-sœur n'en ont jamais parlé ouvertement et le poids d'une double culpabilité les empêche de vivre : avoir souhaité la mort de Tanilo, et avoir eu une relation adultère à son insu et presque sous ses yeux. Cette libération verbale, cette confession, n'atteint aucun auditeur intradiégétique ; comment pourrait-il en être autrement sans que les conséquences soient dramatiques pour toute la famille?

Dans le conte " Anacleto Morones », Lucas Lucatero dit qu'il a tué son maître, Anacleto Morones ; il se le dit en son for intérieur, et se garde bien de le révéler aux dévotes qui ont envahi sa maison dans le but de trouver un témoin des actes miraculeux de leur saint.

Le personnage narrateur de «La herencia de Matilde Arcángel » laisse échapper sa rancune envers la femme qu'il aimait, avec laquelle il s'était fiancé, et qui l'avait quitté pour son meilleur ami. 
Le monologue intérieur dans l'œuvre de Juan Rulfo : une tromperie cathartique

Ces contes sont donc le lieu d'expression privilégié d'une parole qui était vouée à n'être jamais formulée, puisqu'elle contient des révélations accablantes impliquant l'honnêteté des autorités, ou la culpabilité de certaines personnes dans des affaires oubliées. Les monologues n'ont qu'une vertu cathartique. Tous ces personnages doivent avant tout s'avouer la vérité pour pouvoir par la suite la propager. Or cette acceptation de la vérité passe tout d'abord par une acceptation de soi, rendue impossible par le regard absent de l'autre.

Le monologue revêt aussi cette même fonction dans l'organisation du roman. Bien entendu, au premier abord, le dialogue semble être un modèle beaucoup plus englobant. L'énonciation provient de la tombe que partagent Juan Preciado et Dorotea, et c'est leur dialogue qui génère tout le roman. Mais, il vaudrait mieux parler de «pseudo-dialogue». En effet, l'échange entre ces deux personnages est très limité ; il se réduit aux récits a priori indépendants de fragments d'histoire. Juan Preciado explique ce qui l'a amené à Comala et ce qui lui est arrivé jusqu'à sa mort ; Dorotea éclaircit quelques périodes de la vie de Pedro Páramo. Les moments où ils se répondent l'un l'autre sont extrêmement rares, et leurs propos restent souvent dans le vide :

« [Dorotea] - Yo. Yo vi morir a doña Susanita.

[Juan Preciado] - ¿Qué dices, Dorotea?

- Lo que te acabo de decir. $»^{2}$

Autrement dit, la teneur de leur échange discursif n'est pas fondamentale. Ce fragment de dialogue est d'ailleurs représentatif de tous les autres, par le manque de communication entre les personnages dont il témoigne. Chaque personnage est enfermé dans son propre monde, et aucun écho n'est possible entre ces espaces irrémédiablement cloisonnés. Le cacique regrette de ne jamais être parvenu à pénétrer le monde de son unique amour, Susana, qui est enfermée dans la folie, et cette situation est le lot de chacun. Donis et sa sœur, Dolores Preciado et Pedro Páramo ou Miguel et Pedro Páramo sont-ils jamais parvenus à s'entendre ?

${ }^{2}$ Idem, p. 145. 
C'est la raison pour laquelle le monologue est le véritable élément structurant de cette œuvre. L'œuvre de Juan Rulfo est construite comme un concert polyphonique et harmonique. Par exemple, les différentes versions de la mort de Miguel sont juxtaposées; Fulgor, Eduviges et les fossoyeurs en donnent une vision particulière, sans que leur volonté soit celle de se contredire l'un l'autre. Chacun raconte simplement sa propre expérience ou sa propre perception des événements. Chaque fragment épouse un point de vue précis qui correspond toujours à celui d'un personnage. De fait, Pedro Páramo apparaît comme un recueil de monologues qui, assemblés, retracent la véritable histoire de Comala, de la naissance de Pedro Páramo à la mort de Juan Preciado.

C'est bien la même fonction qu'a le monologue dans les contes. En donnant la parole aux minorités économiques et culturelles, Juan Rulfo se joue de la censure et des relations de pouvoir. Certes les paysans de « Nos han dado la tierra » garderont leurs terres arides, certes le vieil Esteban sera jugé coupable d'homicide volontaire, certes Juvencio Nava sera exécuté. Mais ils ont tous eu le temps de dévoiler, au moins en partie, une autre version des faits différente de celle qui est diffusée par le pouvoir. La polyphonie qui découle de la division de la voix narrative est le contre pouvoir le plus efficace face à une parole univoque et autoritaire. En outre dans le roman, la mort est le véritable lieu de l'énonciation. Comme dans la tradition gréco-latine, la mort est le berceau de la vérité. Le passage en enfer de certains vivants est un parcours initiatique qui vise à dévoiler à l'homme le sens de son existence ou certaines vérités. La localisation de l'énonciation de Pedro Páramo dans l'enfer de Comala permet de redonner à la parole l'autonomie dont elle a besoin pour diffuser la vérité historique. Or, la large prédominance du monologue sur le dialogue dans l'œuvre rulfienne s'explique peut-être par le fait que ces paroles multiples et complémentaires se posent comme étant les différentes pièces qui, assemblées, construisent l'unique vérité qui ne peut être contredite dans un dialogue. Nous y reviendrons. 
Le monologue intérieur dans l'œuvre de Juan Rulfo : une tromperie cathartique

Pourtant le monologue prend souvent, chez Rulfo, l'apparence d'un faux dialogue et, en ce sens, il n'est plus seulement le signe d'une organisation sociale perverse mais d'un malaise beaucoup plus individuel.

En effet, dans sept contes et cinq fragments du roman, le personnage qui parle s'adresse à un interlocuteur invisible. Trois situations sont possibles.

Le monologue classique, qui correspond à une personne qui parle seule. Cela arrive à Fulgor Sedano aux fragments vingt et trente et un, et au padre Rentería au fragment trentequatre. C'est la structure de base de référence. Les deux personnages cités se disent en leur for intérieur ce que le respect hiérarchique pour le premier, ou les règles religieuses pour le second, empêchent de dire tout haut. Pour les mêmes raisons, le personnage-narrateur du conte « La Cuesta de las Comadres » est obligé de parler avec un mort, car ce dernier ne lui avait pas laissé la possibilité de dire la vérité tant qu'il était en vie. Le monologue intérieur est donc généré par la prohibition de la liberté d'expression.

Parfois la personne qui parle seule semble s'adresser à un interlocuteur indéfini qui pourrait lui répondre. Ainsi Susana San Juan invoque-t-elle une deuxième personne qui n'existe que dans ses rêves, et, au milieu d'une longue séquence nostalgique, elle dit :

«Mi madre murió entonces.

Que yo debía haber gritado ; que mis manos tenían que haberse hecho pedazos estrujando su desesperación. Así hubieras tú querido que fuera. ¿Pero acaso no era alegre aquella mañana ? $»^{3}$

Nous ne savons pas qui est ce « tú » à qui elle s'adresse. Peut-être Justina à qui elle posera des questions un peu plus tard ? Peut-être aussi son propre « je » qui ne parvient pas à se construire étant donné l'absence de l'Autre ? L'identité du sujet ne peut se construire qu'en inventant une altérité virtuelle. Ce «tú » invoqué convoque à la fois l'interlocuteur et, par son intermédiaire, le locuteur. Le monologue, prenant quelques caractéristiques du dialogue, est une tentative, la seule possible, pour construire l'identité du sujet. Nous

${ }^{3}$ Idem, p. 96. 
retrouvons cette même instance supérieure indéfinie dans les monologues intérieurs d'autres personnages, dont la santé mentale n'est pas en cause. L'éclopé, qui a été mutilé par Pedro Páramo après le meurtre de son père, s'adresse à un « ustedes » :

«Él no tuvo intenciones de matarme. Me dejó cojo, como ustedes ven, y manco si ustedes quieren. Pero no me mató. ${ }^{4}$

Or, c'est bien un défunt qui parle depuis l'au-delà. Il n'est pas envisageable que le « ustedes » du monologue de l'homme torturé fasse allusion à Dorotea et Juan Preciado ; en effet, rien ne nous indique qu'il y ait communication entre les tombes.

Nous retrouvons ce même pronom dans le monologue du personnage-narrateur du conte "La herencia de Matilde Arcángel ». Ce conte est construit de façon à faire croire au lecteur qu'il en est le destinataire. Le narrateur fait plusieurs appels à un interlocuteur, qui n'est pas présent dans l'histoire, et qui laisse penser que le narrateur joue le rôle d'un conteur s'inventant un public qui deviendrait le témoin de son histoire. Il est très méthodique - voire didactique - dans sa façon de raconter :

«[...] Sin embargo, habrá que decirles antes quién y qué cosa era Matilde Arcángel. Y allá voy. Les contaré esto sin apuraciones. Despacio. Al fin y al cabo tenemos toda la vida por delante. $»^{5}$

Cette supercherie est fréquente chez Rulfo. Dans Pedro Páramo, le lecteur ne croit-il pas, jusqu'à la moitié du roman, que Juan Preciado s'adresse à lui ? La technique est la même dans les contes, à la différence près que dans ces derniers, elle fonctionne jusqu'à la fin sans que le doute puisse être levé. Dans «La herencia de Matilde Arcángel », jusqu'à la fin, le récit est ponctué d'expressions relevant de la captatio benevolentia :

« Ustedes saben, uno es arriero. Por puro gusto. »

${ }^{4}$ Idem, p. 100.

5 Juan RULFO, El llano en llamas, edición de Carlos Blanca Aguinaga, Madrid : Cátedra, 1994, 181p., p. 161. 
Le monologue intérieur dans l'œuvre de Juan Rulfo : une tromperie cathartique

« No podría yo contarles los detalles de porqué y cómo se desbocó el caballo [...]. »

"Ya les conté ... », "Ya les dije... », " Como les dije antes... ${ }^{6}$

Le narrateur va même jusqu'à s'associer à son destinataire dans une constatation d'ordre général :

«Y ustedes y yo y todos sabemos que el tiempo es más pesado que la más pesada carga que puede soportar el hombre. ${ }^{7}$

Il est plus que probable que cet homme, comme la personne torturée du roman ou Susana San Juan, se soit inventé un auditeur qui n'existe absolument pas, mais qui donne sens à ses propos car, sans autrui, la parole n'a pas de sens. Le vieil Esteban du conte «En la madrugada » parle aussi, dans son soliloque, à un « usted» mystérieux. Nous pouvons supposer, par déduction - puisque rien n'est absolument clair chez Rulfo - qu'Esteban est enfermé en prison et qu'il ressasse le semblant de procès auquel il a eu droit. L'altérité est nécessaire à la parole qui, à son tour, est indispensable à la constitution de l'individu social; là est le problème des êtres solitaires de El llano en llamas et de Pedro Páramo.

Ainsi, dans cette œuvre, nous trouvons une dernière catégorie de monologues : ceux qui font appel à une tierce personne qui, tout en étant clairement désignée, soit n'est pas physiquement présente dans l'espace-temps du locuteur, soit reste silencieuse et cachée.

Nous supposons que Melitón et son complice racontent la venue du gouverneur à un auditoire qui n'avait pas pu y assister. Peut-être même pouvons-nous être plus précis. Lorsque celui qui mène la discussion s'adresse à son public en disant :

${ }^{6}$ Idem, p. 161, 162, 163. C'est nous qui soulignons.

${ }^{7}$ Idem, p. 164. 
« Todos ustedes saben que nomás con que se presente el gobernador, con tal de que la gente lo mire, todo se queda arreglado ${ }^{8}$,

il instaure une connivence avec ce « ustedes » qui, comme lui, n'est pas dupe des manœuvres du gouverneur. Un regard critique se pose alors sur le comportement et le discours du représentant de l'état. Mais nous n'avons jamais la preuve que ce consensus de voix existe bien. Ce « ustedes » reste un interlocuteur invisible.

De la même façon, dans "Luvina » et «El hombre », chaque personnage-narrateur s'adresse à un « usted » dont nous ne voyons jamais le visage. Le premier est attablé à un bar avec un autre homme qui ne lui répond jamais rien, reste dans l'ombre, et ne boit même pas ses verres de bière. Le second, qui, pour avoir nourri par ignorance le meurtrier de la famille Urquidi, est accusé de complicité de meurtre, raconte son histoire au juge. Il essaie de prendre sa propre défense en s'adressant à un « usted » qui représente le juge qu'il doit peut-être avoir en face de lui, mais qui reste muet jusqu'à la fin du conte. Nous ne connaissons les chefs d'accusation que par l'intermédiaire de l'accusé lui-même, qui reprend les propos du juge. Mais dans tous les cas que nous venons de citer, il n'est pas certain que le destinataire des monologues soit effectivement présent. Peut-être ce ne sont là que des situations purement imaginaires, entièrement créées par un individu dont l'identité est en crise.

L'œuvre de Juan Rulfo conjugue donc un nombre conséquent de monologues organisés de façons différentes. Deux commentaires s'imposent face d'une part à la profusion de ce type de présentation d'un récit, d'autre part à l'interpellation d'un interlocuteur imaginaire qui donne aux monologues rulfiens leur couleur propre.

L'influence que peut avoir un cacique tout-puissant sur une population déjà fragilisée par la misère et les maladies est considérable. L'organisation sociale instaurée par le caciquisme accentue l'égoïsme, l'isolement, et donc la vulnérabilité de l'être. Le dialogue, qui implique une réciprocité

${ }^{8}$ Idem, p. 152. 
Le monologue intérieur dans l'œuvre de Juan Rulfo :

dans les rapports humains est inconcevable dans une telle situation. Le fait d'avoir perpétué cette construction sous forme de monologue dans l'au-delà n'est pas le signe d'un échec. Juan Rulfo n'est pas un révolutionnaire dont l'intention serait de refaire le monde. Le passé ne peut pas être modifié et même dans un contexte imaginaire il ne doit pas l'être. El llano en llamas et Pedro Páramo sont des littératures du souvenir. Elles restituent la mémoire d'un peuple le plus fidèlement possible. Pour cela il n'est rien de mieux que de donner la parole à tous les acteurs de l'histoire. La vérité historique ne naît ni du dialogue ni de la lutte; elle est. C'est en ce sens que seul le monologue convient car il permet la polyphonie et donne naissance à une parole libre alors que le dialogue est toujours contraignant. La vérité historique n'émane pas d'une seule voix mais de l'ensemble des voix de ces «hommes sans histoire » comme disait Unamuno.

En outre, l'altérité imaginaire qui se construit dans le monologue ne peut être que difforme, puisqu'elle émane d'un sujet qui est lui-même en difficulté. Cette présence floue de l'autre dans le monologue est singulière. Elle manifeste un malaise du sujet perceptible en de nombreux autres endroits du texte rulfien. Pourquoi faire apparaître un éventuel interlocuteur et le laisser dans l'anonymat et le silence ? Si nous nous en tenons au niveau diégétique, cette tendance du texte est difficilement explicable. Elle perpétue une sorte de censure tout en rendant possible une certaine forme d'expression. La situation est ambiguë et nous conduit à la définition d'une stratégie narrative, elle, parfaitement claire.

Juan Rulfo, dans ses contes et plus encore dans son roman, nous propose un texte scriptible, selon la terminologie barthienne. Le lecteur doit prendre une part active dans la lecture et même dans l'écriture de ce texte qui n'est pas univoque et qui propose plusieurs sens de parcours. Pedro Páramo est construit comme un puzzle formé de plusieurs fragments qui ne se suivent pas dans un ordre chronologique. $\mathrm{Si}$ le lecteur baisse la garde, il se perd dans ce labyrinthe textuel.

Le monologue participe grandement de cette complexité : tout d'abord il fait coexister plusieurs - voire 
toutes - les versions d'un même événement, lesquelles se complètent toujours bien qu'étant parfois très différentes. Il appartient donc au lecteur de reconstruire le référent historique. Le lecteur est le seul à pouvoir le faire puisqu'il est le seul à avoir une vision globale de l'histoire. Ensuite, le monologue rulfien est un dialogue avorté : il crée des interlocuteurs qui restent muets et invisibles. Il oriente le lecteur vers une fausse route et l'oblige à de perpétuels va-et-vient pour retrouver la bonne direction. C'est là une tendance commune à ce texte "totalisant », qui, en voulant donner à voir le monde dans sa globalité - en ne censurant aucun point de vue - trompe souvent son lecteur. Il faut du recul par rapport à la lecture pour pouvoir comprendre - cumprehendere, «prendre ensemble »- ce texte. Syntaxiquement et stylistiquement, tous les sens sont permis. L'errance est donc préalable à la découverte du ou des sens.

Le monologue joue donc un rôle fondamental dans la définition de l'œuvre rulfienne. En effet il participe à la stratégie, avant tout narrative, qui consiste à faire intervenir le lecteur dans l'écriture de l'œuvre. Rien n'est donné d'avance, les sens - complémentaires, parallèles ou divergents - se construisent au fur et à mesure de la lecture et de la relecture. Le monologue permet aussi de tromper la censure en donnant naissance à un texte polyphonique.

Le monologue est donc à la croisée de tous les chemins qui mènent aux sens de l'existence, au croisement entre l'intrahistoire et l'Histoire, au cœur de la vérité que cherche Juan Preciado. 